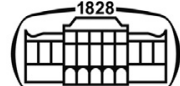

AKADÉMIAI KIADÓ

Journal of Behavioral Addictions

9 (2020) 2, 363-370

D01:

$10.1556 / 2006.2020 .00041$

(c) 2020 The Author

\section{FULL-LENGTH REPORT}

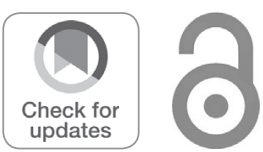

\title{
Positive play and its relationship with gambling harms and benefits
}

\author{
PAUL DELFABBRO ${ }^{1 *}$ (1), DANIEL L. KING ${ }^{2}$ and \\ NEOPHYTOS GEORGIOU ${ }^{1}$ \\ ${ }^{1}$ School of Psychology, University of Adelaide, Adelaide, Australia \\ ${ }^{2}$ College of Education, Psychology, \& Social Work, Flinders University, Bedford Park, Australia
}

Received: February 3, 2020 • Revised manuscript received: May 6, 2020; May 24, 2020 • Accepted: May 24, 2020 - Published online: June 6, 2020

\section{ABSTRACT}

Background: Engagement in responsible or 'positive play' strategies is known to be negatively associated with problem gambling, as indexed by measures such as the Problem Gambling Severity Index (PGSI). Less is known about whether positive play is associated with reduced harm or a greater ability to enjoy the recreational benefits of gambling. Aims: This study investigated the relationship between positive play and gambling harm after controlling for PGSI scores and whether positive play moderated the relationship between PGSI scores and harm. It also examined whether positive play was related to perceived benefits associated with gambling. Methods: The study utilised an online panel sample of 554 respondents who completed a survey that included the PGSI, measures of gambling harm drawn from Browne et al. (2016), and the newly developed Positive Play Scale (Wood et al., 2019). The study involved predominantly monthly gamblers with higher levels of gambling risk: $23 \%$ problem gamblers; $36 \%$ moderate risk; and $21 \%$ low risk gamblers. Results: The results indicated that positive play was negatively associated with reduced gambling harm. The behavioural Positive Play subscales relating to pre-commitment and honesty and control explained additional variation in harm after controlling for PGSI scores. Higher levels of positive play also moderated and reduced the relationship between the PGSI and gambling harm. Perceived benefits were, unexpectedly, found to be higher in problem gamblers and negatively related to positive play. Conclusion: Behavioural measures of positive play appear to be useful moderating factors in understanding the relationship between problem gambling and harm. Higher-risk gamblers appear to experience both greater costs as well as benefits from gambling, which likely reflects a stronger personal need to engage in the activity.

\section{INTRODUCTION}

The majority of people who gamble do so without experiencing any gambling-related harm (Browne et al., 2016). Estimates suggest that around 3-4\% of the adult population in Western countries experience moderate to serious problems associated with gambling (Calado \& Griffiths, 2016; Williams, Volberg, \& Stevens, 2012). Other gamblers generally seem to be able to maintain their gambling at safe levels and do not spend beyond their means or significantly compromise other life commitments. Even when harm has been studied in lower risk populations (e.g. Browne et al., 2016) the harms reported appear principally related to the preferencing of gambling over other activities, with only rare reports of serious harm (e.g. bankruptcy, loss of relationship). This argument holds, notwithstanding, the recognition that regular participation in some forms of gambling (e.g. electronic gaming machines) can often be associated with quite high rates of problem gambling (estimates in the order of 15-20\% have been reported) (Dowling, Smith, \& Thomas, 2005; Livingstone \& Woolley, 2007).

Much of the focus of gambling research has been upon the risk factors associated with problem gambling. In other words, what characteristics, situations, activities or behaviours make problematic gambling more likely to emerge? Many of the behavioural risk factors are, for example, well-known and include: chasing losses; spending more time and money than one can afford; using credit to gamble; selling assets; being secretive and trying to use skill 
and strategy when the outcome is entirely (or largely) determined by chance (Petry, 2005). However, until recently, not much was known about what factors enabled the majority of gamblers to avoid developing serious harm. Accordingly, there have been a number of attempts to capture what might be considered protective or responsible gambling behaviours or 'safe gambling practices' (Hing, Russell, \& Hronis, 2018). Some examples of these safe gambling practices include sticking to a budget, diversifying their leisure activities, avoiding gambling when they are feeling depressed, as well as not using credit for gambling. A measure that has recently been used in a number of major studies around the world (e.g. in Canada) is the Positive Play Scale (PPS) (Wood, Wohl, Tabri, \& Philander, 2017). According to the authors, positive play has a number of elements. People who gamble in a way that minimises harm generally remain in control of their gambling; they set a budget in advance (or engage in a form of pre-commitment); have realistic beliefs about gambling (e.g. they avoid common biases associated with gambling such as they are 'due for a win'). Positive players also take responsibility for their actions by recognising that they have some ownership over the decisions that can lead to excessive expenditure. The term 'positive', in this context, does not refer to positive reinforcement (i.e. enjoyment or excitement) from gambling which are experiences which could potentially exist with or without the presence of the behaviours and attitudes described in the PPS.

According to Wood et al. (2017), positive play is not merely an absence of risky or problematic play, but a set of behaviours which can exist independently of other behaviours. Thus, while avoiding the use of a credit card or not spending more than one can afford could be seen as being something akin to the reverse of a risk factor, there are other aspects of positive play, e.g. staying in control, setting a budget, being realistic about one's chances which are not usually captured by common screening tools (e.g. the Problem Gambling Severity Index (PGSI); Ferris \& Wynne, 2001). They argue that measurement of positive play is useful for two reasons. First, it switches the focus of analysis towards the types of behaviour which are likely to be amenable to change across a wider population of gamblers. Second, positive play focuses on behaviours which are likely to be more common and measurable than risky behaviours that tend to be rarely endorsed by non-problem gamblers. In their validation of the PPS, Wood et al. made several observations. Positive play is negatively related, but only modestly related, to PGSI scores. In other words, as was hypothesised by Wood et al. (2017): (a) people who experience problems with gambling tend to score lower on the PPS and (b) positive play appears to share only modest variance with PGSI, which supports the view that it is measuring something other than merely the reverse of high risk behaviour.

Several questions have not, however, been investigated. The first is whether positive play might play some role in the prevention of harm. The PGSI is, for example, not a harm measure in that it contains few items on this topic, so there may be value in understanding whether engagement in positive play is related to reduced harm. Another related question relates to the benefits associated with gambling. It is well-documented that people gamble in the hope of receiving benefits from gambling. Many of these motivations or expectations are captured by a range of motivational measures (Abarbanel, 2014; Flack \& Stevens, 2019; Nower \& Blaszczynski, 2010; Shinaprayoon, Carter, Goodie, 2017). Such motivations include the desire to win money, to relax or escape from problems, social motives, or a desire to apply skill or feel important. As Ben-Tovim, Esterman, Tolchard, and Battersby (2001) point out, one of the characteristics of problem gamblers (PGs), and identified using a specific question in their Victorian Gambling Screen (VGS), is that PGs find it increasingly difficult to gain the same satisfaction from gambling as might have been so previously. Thus, another potentially useful aspect of positive play is to examine whether engaging in this style of play (which is largely defined in terms of responsible gambling behaviours and attitudes) allows for the greater realisation of the benefits of gambling. In everyday terms, does positive play enable people who gamble to keep their gambling more 'recreational'?

\section{The present study}

The principal aim of this paper, therefore, was to extend existing analyses of positive play to investigate several questions of interest. The first was to investigate whether engagement in more positive play is associated with reduced gambling harm and the extent to which the Positive Play Subscales are able to predict harm after controlling for problem gambling scores (as indexed by the PGSI). The second was to investigate whether those who score higher on the PPS measures experienced increased benefits from gambling. A third analysis was to examine whether the relationship between problem gambling and harm is moderated by positive play. Measures such as the PGSI focus largely on behaviours associated with excessive gambling (e.g. spending more than one can afford, chasing or borrowing money). Thus, it would be useful to know to what extent is the relationship between the PGSI and harm influenced when gamblers engage in various levels of responsible gambling. It was hypothesised that positive play would be negatively associated with PGSI scores and gambling harm and positively associated with greater gambling-related benefits, and that the relationship between the PGSI and gambling harm would be reduced when people reported greater positive or more responsible play.

\section{METHOD}

This paper is a companion piece for XXX (2020, submitted) which focuses on the refinement of harm measures. The two papers draw upon the same data-set, but with different measures (apart from the PGSI and aggregate harm count). 
The methodology is briefly summarised again in this paper to aid the reader.

\section{Participants}

A total of 554 adult gamblers, all aged 18 years or older, $(\mathrm{M}=64 \%, \mathrm{~F}=34 \%, 2 \%$ another classification) completed an online survey through the online panel provider (Prolific). The advertising indicated that participants had to gamble at least once per month on activities other than lotteries. Responses were obtained from those who had gambled in the last 12 months on activities other than lotteries and were principally sampled from: North America (40\%); the UK (16\%); Australia (7\%) as well as other countries (37\%). A total of 401 (72\%) were aged $18-34$ years, another $102(18.2 \%)$ were aged 35-44 years and the remainder (52 or $9.4 \%$ ) were aged 45 and older. The majority were in some form of paid employment: 285 (51.4\%) full-time, $137(24.7 \%)$ in part-time or casual work. There were 128 (23\%) problem gamblers; 198 (36\%) moderate-risk gamblers; 119 (22\%) low risk gamblers and 109 (20\%) recreational gamblers as based on the PGSI classification (see below).

\section{Procedure}

Advertisements were posted on the Prolific portal and participants who had gambled on non-lottery products were eligible to participate. The mean duration of the survey was 16 minutes and participants were paid a fee based on completion. The final sample comprised 554 gamblers after incomplete, aberrant (e.g. bankrupt due to gambling and PGSI Score of 0) or ineligible participants were excluded (e.g. people who did not gamble at all). Almost 200 cases either attempted (150) or completed surveys (50) had to be excluded.

\section{Measures}

Demographics and gambling participation. Each respondent indicated their gender, age, work status, living status, and financial status as based on whether they could raise the equivalent of $\$ 2000$ Australian in an emergency (US, UK and Euro equivalents provided). They also rated how often they gambled on various activities: casino and card games; electronic gaming machines; sports; racing, lotteries, scratch tickets and other activities. These responses could range from $1=$ Never to $5=$ Weekly or more often.

Problem Gambling Severity Index (PGSI). The PGSI (Ferris \& Wynne, 2001), the well-used 9-item screening tool was completed by all participants. Items were scored using a 4-point response scale, where $0=$ Never, $1=$ Sometimes, 2 $=$ Most of the time, $3=$ Almost always and was administered with a last 12-months time-frame. Scores of 0 indicate recreational gambling; $1-2=$ low risk gambling, $3-7$ is classified as moderate risk gambling and scores of 8-27 (maximum) indicate problem gambling. These cut-offs revealed that 128 (23\%) were problem gamblers; 198 (37\%) were moderate-risk gamblers; 119 (22\%) were low risk gamblers; and, 109 (20\%) were recreational gamblers. The Cronbach's alpha was .88 for this sample.

Gambling-related harm. The study drew upon a list of detailed items developed by Browne et al. (2016). These fell into several categories that captured mild harms as well as the most severe harms that might arise from gambling. These included: financial (reduced savings due to gambling - Becoming bankrupt); Work/Study (being late for work Losing a job); Health (Reduced sleep - Emergency room); Psychological (Regret - Suicide attempts); Social (Spending less time with important people - Separations); Other (Mostly deviant behaviours such as crimes and taking money). We used a total of 63 harm items and scored them using a method recommended by Blaszczynski, Anjoul, Shannon, Keen, Pickering, and Wieczorek (2015). In this method, each harm item was presented in a general form first, e.g. In the LAST 12 months 'Have you felt hopeless? with a 5 point rating scale: $1=$ Not a problem, $2=\mathrm{A}$ minor problem, $3=$ A moderate problem, $4=$ A major problem, 5 $=\mathrm{A}$ very serious problem. Respondents who gave a response of at least 2 were then asked to rate the harm on a second scale: "My feelings of hopelessness were": $1=$ Not caused by my gambling, 2 = Slightly caused by my gambling, 3 = Moderated caused by my gambling, $4=$ Mostly caused by my gambling and $5=$ Totally caused by my gambling. In this paper, we worked out how many of the 63 items each person endorsed using the 'moderate problem' scoring method. This was based on responses to the pair of questions asked for each harm item. For each harm item, this required that the person: (a) rate each harm as at least a moderate problem on the first question, and then (b) indicate that the problem was at least moderately caused by their gambling. We then totalled all items that met this threshold for a given individual to yield a total harm score out of 63 .

The Positive Play Scale (PPS). This measure was developed by Wood et al. (2017) and comprises four separate subscales. Two of the scales are behavioural and the other two are attitudinal. The first behavioural subscale Honesty and Control (with 3 items) is scored on a 7-point Likert scale from 1 to 7 where $1=$ Never and $7=$ Always (subscale range 3-21). A sample item includes: "I feel in control of my gambling behaviour". The second behavioural scale, Precommitment (which relates to sensible time and money management) has 4 items which are scored the same way (subscale range 4-28), e.g. "I considered the amount of money I was willing to lose before I gambled" or "I only gambled with money that I could afford to lose". The first attitudinal subscale, Personal Responsibility, has 4 items and these are scored $1=$ Strongly disagree to $7=$ Strongly agree (subscale range 4-28). An example item is "I should be aware of how much money I spend when I gamble". The second attitudinal scale, Gambling Literacy, has 3 items scored the same way (subscale range 3-21) (e.g. "My chances of winning get better after I have lost"). Each of these subscales is scored so that higher totals indicate more positive or 
'responsible' play. The Alpha Coefficients for all subscales were good to very good: Honesty and Control (0.86); Precommitment (0.90); Personal responsibility (0.86) and Gambling Literacy (0.73).

Benefits of gambling. A 10-item benefits of gambling scale was developed for this study. Participants had to rate each statement on a 5 -point Likert scale from $1=$ Strongly disagree, $2=$ Disagree, $3=$ Neutral, $4=$ Agree, and $5=$ Strongly agree (range 10-50) with higher scores indicating greater perceived benefits of gambling. A Principal Components analysis using Oblim rotation (SPSS, v25) indicated that all items loaded highly ( 0.6 or higher) on a single factor that explained $47 \%$ of variance (Eigenvalue of 4.67 ). A small second factor only attracted a loading $>0.5$ for one item and had an Eigenvalue of 1.2. A Scree-plot and assessment of the interpretability of the solution indicated that a single factor solution was the best one. The items were chosen in preference to the Gambling Motivations Scale (Shinaprayoon et al., 2017) or Gambling Effects Scale (Flack \& Stevens, 2019) because we were interested in the perceived benefits or outcomes of gambling as opposed to what people might hope to gain from gambling (their motivation). Items included: Gambling makes me: feel happy; keeps my mind active; gets me out of the house and gives me something to do; is exciting for me; gets me out doing things with other people; makes my life more interesting; gives me something to look forward to, as well as "I would miss not being able to gamble if the activity was not available", "I would go out less if there were no gambling activities" and "I would feel unhappy or bored if I could not gamble". The Alpha for the scale was 0.86 , indicating very good internal consistency.

Analytic strategy. The study used one-way ANOVA and Bonferroni tests to compare harm, benefit and positive play scores between the different PGSI groups. Pearson correlations were used to examine the relationship between measures. Hierarchical regression was used to examine whether positive play was able to predict gambling-related harm after controlling for PGSI scores. To test whether having Positive Play strategies moderated the relationship between PGSI scores and harm, a hierarchical regression was again used with the main effects entered on the first step (Model 1) and the interaction of the PGSI and the PPS subscale on Step 2.

\section{Ethics}

The project was approved by the Human Research Ethics Subcommittee in the School of Psychology. Approval Number: 19/86.

\section{RESULTS}

\section{(a) Descriptive statistics and mean comparisons}

Scores on measures of harm (total count of moderate harm), positive play and gambling benefits were compared across the four PGSI groups (Table 1). As previously reported by Delfabbro, Georgiou, and King (2020), self-reported gambling harm was highest in problem gamblers and quite low in the lower risk groups. The scores for three positive play subscales (Honesty and control, Pre-commitment and Gambling Literacy) were, as hypothesised; namely, higher in the lowest risk groups and lowest in problem gamblers. However, personal responsibility scores were higher for problem gamblers. Problem gamblers also reported obtaining more benefits from gambling than the lower risk groups.

\section{(b) Correlations}

A summary of Pearson correlations is provided in Table 2. The results showed that the behavioural measures of positive play (honesty-control and precommitment) are negatively related to PGSI scores and the level of gambling harm. Gambling literacy was also lower when PGSI scores and the total harm count were higher. However, the Person-responsibility scale scores were positively related to problem gambling and harm. This scale was negatively associated with the other Positive Play subscales. Perceived benefits were found to be negatively (although weakly) associated with positive play subscales.

(c) Incremental value of Positive Play as predictor of gambling harm

A hierarchical regression was conducted to investigate whether the PPS was predictive of gambling harm after

Table 1. PGSI group comparisons: M (SD) gambling harm, benefits and positive play subscales

\begin{tabular}{|c|c|c|c|c|c|c|c|}
\hline $\begin{array}{l}\text { Measure } \\
\qquad \text { RG }(n=109)\end{array}$ & $\begin{array}{c}1 \\
\text { Low risk } \\
(n=119)\end{array}$ & $\begin{array}{c}2 \\
\text { Mod risk } \\
(n=198)\end{array}$ & $\begin{array}{c}3 \\
\text { Problem } \\
(n=128)\end{array}$ & $M(S D)$ & $F(3,550)$ & $\begin{array}{c}\eta^{2} \\
M(S D)\end{array}$ & $\begin{array}{l}\text { Post-hoc } \\
M(S D)\end{array}$ \\
\hline Honesty \& Control & $18.0(4.8)$ & $17.5(4.2)$ & $15.8(4.9)$ & $13.8(4.9)$ & $19.4^{* * *}$ & 0.096 & $3-4<1-2$ \\
\hline Pre-commitment & $24.1(8.8)$ & $24.0(5.1)$ & $21.8(6.2)$ & $19.3(6.2)$ & $18.4^{* * *}$ & 0.091 & $3-4<1-2$ \\
\hline Personal Resp & $6.3(4.7)$ & $5.7(2.7)$ & $6.7(4.1)$ & $8.3(4.5)$ & $9.3^{* *}$ & 0.048 & $4>1-3,3>2$ \\
\hline Gambling literacy & $15.3(2.5)$ & $15.4(2.2)$ & $15.0(2.1)$ & $14.0(2.6)$ & $9.5^{* *}$ & 0.049 & $4<1-3$ \\
\hline Gambling benefits & $27.9(7.9)$ & $29.1(6.9)$ & $31.0(7.7)$ & $33.5(6.7)$ & $13.5^{* *}$ & 0.068 & $4>3,3>1-2$ \\
\hline Gambling harm & $0.81(2.3)$ & $0.49(1.3)$ & $2.09(5.3)$ & $7.00(9.9)$ & $33.31 * * *$ & 0.154 & $4>1-3,3>1-2$ \\
\hline
\end{tabular}

$\mathrm{RG}=$ Recreational gambler. Interpretation of post hoc test: $4<1-3$ means that the mean for group 4 was significantly lower than group 1,2 and 3 (in separate post-hoc tests). ${ }^{* *} P<0.01 * * * P<0.001$ 
Table 2. Pearson correlations between principal measures

\begin{tabular}{|c|c|c|c|c|c|c|}
\hline & Benefits & Harm & Hon-control & Pre-commit & Pers Resp & $\begin{array}{r}\text { Gam } \\
\text { Lit }\end{array}$ \\
\hline PGSI & $0.25^{* *}$ & $0.24^{* *}$ & $-0.31 * *$ & $-0.31 * *$ & $0.18^{* *}$ & $-0.20 * *$ \\
\hline Benefits & & $0.26^{* *}$ & $-0.18^{* *}$ & $-0.12^{*}$ & $-0.14^{*}$ & $-0.14^{*}$ \\
\hline Harm & & & $-0.42 *$ & $-0.43 * *$ & $0.26 * *$ & $-0.18^{*}$ \\
\hline Hon-Control & & & & $0.66 * *$ & $-0.48 * *$ & $0.16^{*}$ \\
\hline Pre-commit & & & & & $-0.54 * *$ & $0.24^{*}$ \\
\hline Pers Resp & & & & & & $-0.38 * *$ \\
\hline
\end{tabular}

${ }^{*} P<0.05,{ }^{* *} P<0.01$. Positive play subscales: Honesty-control; Pre-commitment; Personal Responsibility; Gambling Literacy.

controlling for PGSI scores. This analysis helps to determine whether Positive Play is more than a reversal of the types of item included in the PSGI. The PGSI was entered into the model on Step 1 and the PPS subscales on step 2. As indicated in Table 3, both of the behavioural subscales remained significant and negatively associated with PGSI scores. Model 2 explained additional variance above that accounted for by Model 1 (PGSI only). Neither of the attitudinal subscales (Personal responsibility or Gambling literacy) remained significant.

(d) Does positive play moderate the association between problem gambling (PGSI) and harm?

To investigate whether the Positive Play subscales moderated the relationship between PGSI scores and harm, four separate moderation analyses were conducted. These were performed using the Process modification to SPSS (Hayes, 2018). This technique involves testing for the interaction and change in R-squared and then examining the nature of the conditional association between the predictor variable (X: here PGSI) and the dependent measure (Y: here total harm) at each level of the moderator (W: here each Positive Play (PP) scale in turn). The results of these four models are summarised in Table 4. As indicated, all four interaction terms were significant, although the effect for the final model (Gambling literacy) was weak.

Insights into the nature of the moderation are provided in Table 5. Table 5 shows the nature of the association

Table 3. Hierarchical multiple regression: Incremental contribution of PPS subscales to the prediction of total harm after controlling for PGSI scores

\begin{tabular}{|c|c|c|c|c|c|c|}
\hline & \multicolumn{3}{|c|}{ Model 1} & \multicolumn{3}{|c|}{ Model 2} \\
\hline & B & Beta & $t$-value & B & Beta & $t$-value \\
\hline PGSI & 0.57 & 0.42 & $10.8^{*}$ & 0.39 & 0.28 & $7.37^{\star *}$ \\
\hline Hon-control & & & & -0.25 & -0.20 & $3.96^{\star *}$ \\
\hline Pre-commit & & & & -0.22 & -0.22 & $4.24^{\star *}$ \\
\hline Pers Resp & & & & -0.05 & -0.03 & $<1$ \\
\hline Gam Lit & & & & -0.15 & -0.06 & 1.41 \\
\hline$R^{2}$ & & & .175 & & & 0.297 \\
\hline$F D f$ & & & $117.4^{\star * *}$ & & & $\begin{array}{l}46.3^{* * *} \\
(5,553)\end{array}$ \\
\hline
\end{tabular}

${ }^{* *} P<0.01$. ${ }^{* *} P<0.001$. Abbreviations: Problem Gambling Severity Index; Honesty and Control; Pre-commitment; Personal Responsibility; Gambling literacy between the PGSI and harm at $-1 \mathrm{SD}$, the mean and $+1 \mathrm{SD}$ values of the moderator (the relevant PP scale). For Honesty and control and Precommitment, the pattern of results is very similar. When PP is low, there is a positive relationship between PGSI score and harm, but this becomes non-significant when scores on these subscales are higher. For Personal responsibility, the results are quite different. As the gambler's feeling of personal responsibility increases, the relationship between the PGSI and harm remains positive, but the unstandardised coefficient value becomes larger. As Gambling Literacy increases, the value of the unstandardised coefficient becomes smaller, but it still remains significant at all levels of the moderator.

\section{DISCUSSION}

The findings of this study were generally consistent with the conceptual groundwork set out by Wood et al. (2017) in the

Table 4. Hierarchical multiple regression: testing for Positive Play subscale moderation of the relationship between PGSI scores and total harm

\begin{tabular}{ll}
\hline \multicolumn{2}{c}{ Unstandardised } \\
\cline { 2 - 3 } Coefficient & $t$-value \\
\hline
\end{tabular}

Honesty-Control (HC)

PGSI

Hon-control

PGSI $x$ HC

0.33

$6.26^{* * *}$

$-0.40$

$8.51^{* * *}$

$\Delta \mathrm{R}^{2} \mathrm{~F}(1,550)$

$7.56^{\star * *}$

Pre-commitment (PC)

PGSI

Pre-commit

PGSI x PC

$-0.32$

$-0.04$

0.06952 .2

$\Delta R^{2} F(1,550)$

Personal Responsibility (PR)

PGSI

Hon-control

0.27

PGSI $x$ HC

0.05

$6.53^{* * *}$

$8.47^{* * *}$

$5.99^{* * *}$

0.04438 .9

$\Delta \mathrm{R}^{2} \mathrm{~F}(1,550)$

$9.57^{* * *}$

$4.57^{* * *}$

$4.35^{* * *}$

0.02318 .9

Gambling literacy (GL)

PGSI

Pre-commit

PGSI x PC

0.52

$-0.27$

0.05

$9.67^{* * *}$

$2.55^{*}$

$2.37^{*}$

$\Delta R^{2} F(1,550)$

$0.00825 .60^{* *}$

${ }^{\star} P<0.05{ }^{* *} P<0.01 .{ }^{* *} P<0.001$. 
Table 5. Analysis of interactions: Conditional effects at the value of the moderator

\begin{tabular}{lcc}
\hline \multirow{2}{*}{ Mod value } & \multicolumn{2}{c}{ Unstandardized } \\
\cline { 2 - 3 } Honesty and control & coefficients & $t$-value \\
-4.99 & 0.67 & $11.42^{* * *}$ \\
0 & 0.33 & $6.26^{* * *}$ \\
4.87 & -0.012 & $<1$ \\
0 & 0.34 & $6.53^{* * *}$ \\
5.84 & 0.09 & 1.21 \\
Personality responsibility & & \\
-2.75 & 0.35 & $5.29^{* * *}$ \\
0 & 0.50 & $9.57^{* * *}$ \\
4.15 & 0.73 & $10.47^{* * *}$ \\
Gambling literacy & & \\
-2.37 & 0.64 & $9.50^{* * *}$ \\
0 & 0.52 & $9.67^{* * *}$ \\
2.37 & 0.41 & $5.12^{* * *}$ \\
\hline
\end{tabular}

Note: ${ }^{* *} P<0.001$; Mod value $=$ the -1 SD, Mean and +1 SD values of the moderator.

design of the PPS. Higher scores on the behavioural measures of positive play were, as expected, negatively related to PGSI scores, which suggests that lower risk gamblers report using more 'responsible' gambling practices such as setting budgets, being honest about their gambling and staying in control. The general magnitude of the observed correlations was generally similar to those observed by Wood et al. (2017) (-0.32 and -0.38). We also found that responsible gambling behaviours, as captured by the PPS, were negatively associated with gambling harm, which suggests that greater harm is reported by gamblers who report fewer positive gambling behaviours. These PPS subscales were also found to predict gambling harm after controlling for PGSI scores which is conceptually important because it suggests that the PPS is capturing something other than just the antithesis of what is captured by the PSGI. Positive play appears, in its own right, to be a predictor of gambling harm, but in the reverse and expected direction. Another finding consistent with the original conceptualisation was that PPS appears to moderate the relationship between PGSI or problem gambling (predominantly a behavioural measure) and reports of gambling harm. When reported positive play was low (most notably Honesty and Control and Precommitment), the PGSI was a strong predictor of gambling harm, but this relationship disappeared when positive behaviours were reported. What was not expected, however, was that the perceived benefits of gambling were greater in the higher risk gambling groups. In other words, problem gamblers were more likely to report stronger benefits from gambling than the lower risk groups, which is generally inconsistent with the view that they find gambling somehow less satisfying.

This study showed the relationships observed between the subscales of the PPS and problem gambling and harm differed depending on which subscale was considered. Thus, while we obtained the results observed above for the behavioural subscales, the effects were not as strong or in the direction expected for the attitudinal subscales. As would be expected from numerous studies which have investigated the higher rates of erroneous cognitions in problem gamblers (e.g. Lambos \& Delfabbro, 2007; Raylu \& Oei, 2002a, b), problem gamblers scored lowest on gambling literacy. They were more likely to endorse beliefs consistent with the gambler's fallacy or be optimistic about winning money from gambling. However, gambling literacy was not a significant predictor of gambling harm after controlling for PGSI scores. The other subscale, Personal Responsibility was, in fact, positively related to problem gambling and this was confirmed in the moderation analysis. Although counter-intuitive, this is not surprising. Inspection of the items indicates that all are framed in an obligatory sense: "I should be able to" or "It is my responsibility too", and so endorsement of these items by problem gamblers does not necessarily mean that they follow up on these obligations. Instead, problem gamblers may be more likely to interpret the questions in the sense of "I know I should do X, but I am usually not successful in my attempts". Wood et al. (2017) similarly found that this subscale is not related to PGSI in the same way as the others $(r=0.03)$, so our findings similarly suggest that it is the behavioural subscales of the PPS that appear to be most strongly related to problem gambling.

The finding that problem gamblers would report greater benefits is also not entirely inconsistent with other findings. Although Ben-Tovim et al. (2001) found that a reduction in the enjoyment of gambling was a characteristic of problem gambling, findings from motivational studies generally find that problem gamblers have a strong need to gamble. They often endorse motivational items more strongly than other gamblers: a greater need to escape, to win money, to seek excitement or enjoyment (Abbott \& Volberg, 2000; Roy Morgan Research, 1999; Tse et al., 2005), although these motivations can vary by the type of gambling (Abarbanel, 2014; Parke, Williams, \& Schofield, 2019). In a similar vein, what we endeavoured to identify as benefits of gambling can just as easily be categorised as needs to gamble. By this argument, problem gamblers would be people who have a stronger need to gamble. They are known to score higher on measures of boredom proneness and social isolation which would make gambling an attractive distraction. Thus, there may be fundamental challenges associated with capturing the benefits of gambling by measuring it in isolation. Instead, as Blackman, Browne, Rockloff, and Hing (2019) point out, it may be better to focus on the trade-off of costs and benefits. It is not that problem gamblers fail to receive benefits from gambling; it is merely that the costs they entail may outweigh the benefits. However, as they showed (Tasmanian Department of Treasury and Finance, 2018), when people were asked to rate the percentage benefit and cost of gambling, the correlations between the ratings was 0.66 (a large effect). Those who perceived higher costs also perceived higher benefits. The authors did not indicate which gamblers made these ratings, but our findings in this study suggest that this may be due to higher risk and 
problem gamblers' tendency to report both higher benefits as well as costs. Problem gamblers acknowledge the harm, but also, as would be expected in a behavioural addiction, have a strong need to continue to engage in the activity to obtain the experiences which act as reinforcers for it. The fact that we observed small negative relationships between positive play and benefits is likely to be explained by these other effects. Problem gamblers report higher benefit scores, but lower positive play scores, so that reporting benefits from gambling does not necessarily imply that a person is gambling 'more safely' or 'positively'.

\section{Limitations}

It is important to acknowledge that this study was based on a self-report survey so that one can never be certain that all respondents were honest or always logically consistent in their responding. The design was also cross-sectional so that it is not possible to determine the causal relationships between variables, e.g. whether lower PGSI scores or higher positive play scores might have some other common antecedent factor. The data were also collected from an international panel so that the findings cannot be generalised back to specific jurisdictional populations, but should be treated as indicative of what might occur if the study were replicated in a single population. Nevertheless, the study used a sufficiently powered analysis with over 500 participants and demonstrated some convergent validity and reliability in the findings, e.g. the PGSI and harm results were related to the PPS in same direction and PPS -PGSI correlations were similar to Wood et al. (2017).

\section{Conclusions}

This study confirms the value of using the PPS as a useful and brief psychometric measure to capture responsible gambling behaviours. The behavioural subscales in particular appear to moderate the relationship between problem gambling behaviours and reports of gambling harm. Positive play does not appear to be merely the antithesis of problem gambling measures, but appears to provide an independent contribution to the explanation of gambling-related harm, principally because it captures key elements such as control and pre-commitment which are not constructs which are directly measured (as a deficit) in widely used measures such as the PGSI. It is not clear, however, whether attitudes towards responsibility necessarily translate into responsible behaviour. Many problem gamblers appear to know what they should be doing to make their gambling safer, but there is a disjuncture between what they believe in relation to 'responsible behaviour' and what they do in practice. These findings have potential implications for policies relating to harm minimisation. It appears that problem gamblers do not necessarily lack the knowledge about the potential harms associated with their behaviour. Instead, they have difficulties in modifying their behaviour. This supports the view that harm minimisation measures designed to moderate gambling behaviour (e.g. limits on the ability to spend money very quickly) and practical skills training may be more useful than merely providing information. Although encouraging gamblers to recognise the consequences of their own behaviour is important, effective harm minimisation will often require support to translate this perception into tangible behaviours. An emerging literature relating to "safe gambling practices" (Hing, Browne, Russell, \& Rockloff, 2019) is potential instructive in this regard and includes measures such as: avoiding credit; not gambling when depressed or anxious; and, using appropriate money management strategies. Positive play may be a useful way to identify individuals who could be identified as having strengths that would make them more inclined to adopt a pattern of gambling involvement that it less likely to incur harm.

Funding sources: This paper was funded independently with no support from government, industry or party external to the University.

Authors' contribution: Survey design (DK, PD, NG); Data collection and data preparation (NG); Data analysis (PD); paper draft (PD); editing and review (DK).

Conflict of interest: None to declare.

\section{REFERENCES}

Abarbanel, B. L. (2014). Differences in motivational dimensions across gambling frequency, game choice and medium of play in the United Kingdom. International Gambling Studies, 14, 472-491.

Abbott, M., \& Volberg, R. (2000). Taking the pulse on gambling and problem gambling in New Zealand: A report on phase one of the national prevalence study. Wellington: Department of Internal Affairs.

Ben-Tovim, D., Esterman, A., Tolchard, B., \& Battersby, M. (2001). The Victorian gambling screen. Report prepared for the Gambling Research Panel, Melbourne, Victoria.

Blackman, A., Browne, M., Rockloff, M., \& Hing, N. (2019). Contrasting effects of gambling consumption and gambling problems on subjective wellbeing. Journal of Gambling Studies, 35, 773-792.

Blaszczynski, A., Anjoul, F., Shannon, K., Keen, B., Pickering, D., \& Wieczorek, M. (2015). Gambling harm minimisation report. Sydney: Office of Liquor, Gambling and Racing, NSW.

Browne, M., Langham, E., Rawat, V., Greer, N., Li, E., Rose, J., Rockloff, M., Donaldson, P., Thorne, H., Goodwin, B., Bryden, G., \& Best, T. (2016). Assessing gambling-related harm in Victoria: A public health perspective. Melbourne: Victorian Responsible Gambling Foundation.

Calado, F., \& Griffiths, M.D. (2016). Problem gambling worldwide: A systematic review of empirical research (2000-2015). Journal of Behavioral Addictions, 5, 592-613.

Delfabbro, P. H., Georgiou, N., \& King, D. L. (2020). Measuring gambling harm: The influence of response scaling on estimates and the distribution of harm across PGSI categories. (Revised manuscript submitted). 
Dowling, N., Smith, D., \& Thomas, T. (2005). Electronic gaming machines: Are they the 'crack cocaine' of gambling? Addiction, 100, 33-45.

Ferris, J., \& Wynne, H. (2001). The Canadian problem gambling index: Final report. Ottawa, Canada.

Flack, M., \& Stevens, M. (2019). Gambling motivations: Comparisons across gender and preferred activity. International Gambling Studies, 19, 69-84.

Hayes, A.F. (2018). Introduction to mediation, moderation and conditional process analyses (2nd ed.): A regression approach. New York: Guilford Press.

Hing, N., Browne, M., Russell, A., \& Rockloff, M. (2019). Avoiding gambling harm: An evidence based set of safe gambling practices for consumers. PLOS One, 14, e0224083.

Hing, N., Russell, A.M.T., \& Hronis, A. (2018). A definition and set of principles for responsible consumption on gambling. International Gambling Studies, 18, 359-382.

Lambos, C. \& Delfabbro, P.H. (2007). Numerical reasoning ability and irrational beliefs in problem gambling. International Gambling Studies, 7, 157-172.

Livingstone C., \& Woolley R. (2007). Risky business: A few provocations on the regulation of electronic gambling machines. International Gambling Studies, 7, 361-376.

Nower, L., \& Blaszczynski, A. (2010). Gambling motivations, money-limiting strategies and precommitment preferences for problem gambling versus non-problem gamblers. Journal of Gambling Studies, 26, 361-372.

Parke, J., Williams, R. J., \& Schofield, P. (2019). Exploring psychological need satisfaction from gambling participation and the moderating influence of game preferences. International Gambling Studies, 18, 508-531.
Petry, N. (2005). Pathological gambling: Etiology, comorbidity, and treatment. London: American Psychological Association.

Raylu, N., \& Oei, P. (2004a). The gambling-related cognitions scale. Addiction, 99, 757-769.

Raylu, N., \& Oei, P. (2004b). The Gambling Urges Scale: Development, confirmatory factor analysis, and psychometric properties. Psychology of Addictive Behaviors, 18, 100-105.

Roy Morgan Research. (1999). Sixth survey of community gambling patterns and perceptions. Melbourne: Victorian Casino and Gaming Survey.

Shinaprayoon, T., Carter, N. T., \& Goodie, A. S. (2017). The modified gambling motivations scale: Confirmatory factor analysis and links with problem gambling. Journal of Gambling Issues, 33, 108-135.

Tasmanian Department of Treasury and Finance (2018). Fourth social and economic impact study. Hobart: Tasmanian Government.

Tse, S., Abbott, M., Clarke, D., Townsend, S., Kingi, P., \& Manaia, W. (2005). Why people gamble: Examining the determinants of problem gambling. Prepared for the health research council of New Zealand. Auckland: Auckland UniServices Ltd.

Williams, R. J., Volberg, R. A., \& Stevens, R. M. G. (2012). Population assessment of problem gambling: Methodological influences, standardized rates, jurisdictional differences, and worldwide trends. Report prepared for the Ontario Ministry of Health and Long-Term Care and the Ontario Problem Gambling Research Centre. May 8, 2012. https://www.uleth.ca/ dspace/handle/10133/3068.

Wood, R. T. A., Wohl, M., Tabri, N., \& Philander, K. (2017). Measuring responsible gambling amongst players: Development of the positive play scale. Frontiers in Psychology, 8, Article 227. 